\title{
Robust Optimization Model for Bi-objective Emergency Medical Service Design Problem with Demand Uncertainty
}

\author{
Diah Chaerani $^{1 *}$, Siti Rabiatul Adawiyah ${ }^{1}$, Eman Lesmana $^{1}$
}

\begin{abstract}
Bi-objective Emergency Medical Service Design Problem is a problem to determining the location of the station Emergency Medical Service among all candidate station location, the determination of the number of emergency vehicles allocated to stations being built to serve medical demand. This problem is a multi-objective problem that has two objective functions that minimize cost and maximize service. In the real case, there is often uncertainty in the model such as the number of demand. To deal with the uncertainty on the bi-objective emergency medical service problem is using Robust Optimization which gave optimal solution even in the worst case. Model Bi-objective Emergency Medical Service Design Problem is formulated using Mixed Integer Programming. In this research, Robust Optimization is formulated for Bi-objective Emergency Medical Service Design Problem through Robust Counterpart formulation by assuming uncertainty in demand is box uncertainty and ellipsoidal uncertainty set. We show that in the case of the bi-objective optimization problem, the robust counterpart remains computationally tractable.
\end{abstract}

Keywords: Bi-objective emergency medical service design problem, branch and bound method, demand uncertainty, lexicographic method, mixed integer programming, robust optimization.

\section{Introduction}

According to Zhang and Jiang [1] emergency health services are designed to provide health care to people who need medical assistance quickly and effectively. In the future life, it is hard to be predicted what will happen, maybe in a region can occur disasters, epidemics, accidents or other events that require handling health. This causes emergency health service facilities to be one of the most important public facilities in handling health.

Facilities are a means to facilitate the implementation of functions (KBBI [2]). Emergency health service facilities provide services to emergency medical requests. To be able to provide maximum service, the location of construction of facilities must be considered to reach points of the request. So, it is essential to determine the location of the construction of emergency health service facilities.

According to Mahanani and Rahardjo [3], the selection of the location of an appropriate health facility greatly determines the achievement of overall health services to the community. In conducting climax, selection states the best combination of several characteristics, namely population, location

\footnotetext{
${ }_{1}$ Faculty Mathematics and Science, Mathematics Department, Universitas Padjajaran, Л. Raya Bandung Sumedang km. 21, Jatinangor Sumedang, Indonesia 45361.

Email: d.chaerani@unpad.ac.id

* Corresponding author
}

and distance, form and access. Based on the Government Regulation of the Republic of Indonesia No. 47 of 2016 (see [4]) concerning health care facilities in Article 8 paragraph 3 that the determination of the number and types of Health Service Facilities is carried out by the Regional Government by considering the elements, area, health needs, population size and distribution, disease patterns, utilization, and ability to utilize technology. Then, in article 10 paragraph 1, 2 and 3, it is stated that the Regional Government determines the number of independent Health Workers places based on community needs for health services in one area. The determination of community needs for health services is done through the determination of the ratio of the number of health workers compared to the number population carried out by considering geographical conditions and community accessibility, utility level, and service hours.

Zhang and Jiang [1] have discussed that in emergency health service design problem, some emergency vehicles will be allocated to the facilities constructed. Determining the location of construction of the facility considers the locations of demand, which can be reached from facilities by considering cost factors such as the cost of building facilities, operations and transportation. Operational costs are related to the cost of procuring emergency vehicles at the facility. Transportation costs are related to the distance between facilities and points of the request. Based on this description, the problem of the design of emergency health services includes the problem of location facilities that aim to determine the location of construc- 
tion of facilities by minimizing all related costs. After determining the location of the emergency health care facilities that have been built, the next problem is determining the proportion of requests served by facilities at that location. Then, the next problem is the determination of the number of emergency vehicles allocated to each facility built to support service at each point of request.

Construction of emergency health service facilities not only considers cost factors, but also considers maximum service to demand. Church and Revelle [5], argue that the level of service is measured by the number of requests served within the specified travel standard distance. Therefore, another goal of the emergency health service design problem is to maximize the number of requests served within the standard range. This is the same as minimizing the number of requests that are not served with distances greater than the standard distance. The large number of requests that are not served within the standard distance will be subject to penalty weight costs. This causes the goal to minimize the cost of penalty penalties due to underserved demand. Based on the description above, the purpose of the emergency health service design problem has two objective functions, namely, first minimizing the cost of building facilities, procuring emergency vehicles, transportation, and secondly minimizing the cost of penalty weight due to underserved demand within standard distance. The existence of these two objective functions is then referred to as the bi-objective function.

Research on the issue of emergency health service design examined by Toregas, et al. [6] proposed a Location Set Covering Model (LSCP) with the objective function of minimizing the number of facilities to cover all requests. Then Church and Revelle [5] proposed a Maximal Covering Location Problem (MCLP) that maximizes the number of requests covered by limited facilities. Furthermore, Gendreu, et al. [7] consider multiple coverage models and solutions obtained through taboo search heuristics developed.

Harewood [8] discusses multi-objective problems Maximum Availability Location Problems that adopt the Queuing Probabilistic Location Set Covering Problem approach in determining the location of emergency health services in Barbados by maximizing populations that receive services with predetermined distance and confidence levels and minimize costs over the population. Furthermore, Araz et al. [9] discuss the Maximal Covering Location Multiobjective model to determine the best location with a limited number of emergency vehicles so that the service level is optimal by maximizing the population fulfilled by an emergency vehicle, maximizing population with reserve coverage, and minimizing the total distance travelled from the facility location to each requested point is completed using Linear Lexicographic Programming and Fuzzy Goal Programming. Almeida et al. [10] discuss a multi-objective approach to determining the location of an emergency in an urban area. A discussion on A bi-objective location-allocation problem of temporary emergency stations and ambulance routing in a disaster situation is presented by Moghaddam et al. [11], also discussion on Two-Stage Multiobjective Optimization for Emergency Supplies Allocation Problem under Integrated Uncertainty (see [12]).

Beraldi, et al. [13] overcome uncertainty in the problem of emergency health service design using stochastic programming and some use the probabilistic paradigm to determine the location of emergency health service facilities and the allocation of the number of emergency vehicles. Furthermore, Beraldi, et al. [14] propose formulations and solutions from probabilistic models to determine the optimal location of facilities in a compact emergency system and use stochastic programming to overcome uncertainties with probabilistic constraints with a two-stage framework.

On the issue of emergency health services, demand parameters are one of the most critical parameters. However, in the real case, uncertainty is often found in the request parameters. Therefore, to overcome the uncertainty in the request parameters, an optimization technique is needed to solve it. One of the optimization techniques used to solve uncertainty problems in this problem is Robust Optimization. According to Bental and Nemirovski [15], Robust Optimization is a modelling method combined with a computational tool to process optimization problems where the data is uncertain and only known in the form of a set of uncertainties. In contrast to stochastic optimization which begins by assuming uncertainty with a probabilistic description where uncertainty is a random variable that is known with certainty. Based on the background described, in this paper discussed the Robust Optimization model for Objective Bi-Emergency Health Service Design with uncertain requests to determine the location of construction of facilities, the allocation of the number of emergency vehicles and the proportion of requests served by facilities. Refers to [1], in this problem it is assumed that at the point of construction site no facilities have been built, so that new health facilities will be built to provide services to medical requests around the candidate area of the facilities constructed. The aim is first to minimize the cost of building facilities, procuring emergency vehicles, and transportation, then the second to minimize the cost of penalty weights. 
The bi-objective emergency medical service design problem is formulated as a problem with two objective functions, and mixed integer programming, the Lexicographic Method [16] is to handle the bi-objective function. As we relax first the binary variables, the robust counterpart is formulated in Section 3. To obtain the calculation of the example, the Branch and Bound Method (see [17] is employed. In this paper, the completion of the bi-objective problem in this paper uses the Lexicographic Method [16] which ranks objective functions based on priority. In this paper, the secondary data that refers to journals written by Ndiaye, M., and Alfares, H. [18] is used. The assumptions are the uncertainty lies in the demand parameter, there is no assumed opportunity distribution, candidates for the location of the construction of emergency health care facilities and candidate locations for requests are known, requests can be served by several emergency health care facilities built. Each request on the candidate location request must be served. The parameter assumed in a numerical experiment is the unit of transportation costs, a relatively large number and the weight of uncertain demand. The main aim of this paper is to obtain a Robust Counterpart Optimization formulation that is computationally tractable on the completion of a bi-objective problem model of emergency health service design. Also, to obtain the optimal robust numerical experimental results from the application of Robust Optimization on the completion of the bi-objective problem model of emergency health service design.

In this paper, the discussion focuses on how the robust counterpart of the uncertain bi-objective emergency medical service design problem is obtained. In [1], the discussion of RC with box uncertainty is not discussed, this paper the robust counterpart with box uncertainty is presented. Differs from [1], in this paper we use the box and ellipsoidal uncertainty set as discussed in [19] and [20], where the box and ellipsoidal uncertainty is presented in norm formulations.

\section{Methods}

\section{Bi-objective Emergency Medical Service Problem}

Referring to Zhang and Jiang [1] the issue of emergency health service design has two location points considered, namely the candidate location of the emergency health service facility and the point of location of the request. Emergency health services handle requests from the location of requests. The aim is to determine the location of construction of facilities from all candidate points of facility location, the number of emergency vehicles that are allocated and the proportion of requests handled by each facility for each demand location considering the minimum costs and service level to demand. The forms of costs considered include the cost of building facilities, the cost of procuring emergency vehicles, and transportation costs, while the level of service is measured by maximizing the demand for the same emergency health services by minimizing the number of underserved requests.

Model Bi-objective Problem Emergency Medical Service Design (MBPEMSD) has two objective functions, namely minimizing costs and minimizing unmet demand within a specified travel distance. The specified travel distance is the maximum distance to serve the request $i$ which is denoted by $S$.

The following notation is used in bi-objective emergency medical service design problem model.

\begin{tabular}{|c|c|c|}
\hline \multicolumn{3}{|c|}{ Sets and parameters are } \\
\hline$J$ & : & $\begin{array}{l}\text { set point location of facility construction } \\
\text { with } j \in\{1,2, \ldots, J\}\end{array}$ \\
\hline$I$ & : & $\begin{array}{l}\text { the set of points of demand location } i \in \\
\{1,2, \ldots, I\} \text {, }\end{array}$ \\
\hline$I_{J}$ & : & $\begin{array}{l}\text { the set of demand point greater than } \\
\text { predetermined maximum distance to } \\
\text { handle demand at point } i \text {, i.e. }\left\{i \mid d_{i j}>S\right\}\end{array}$ \\
\hline$f_{j}$ & : & $\begin{array}{l}\text { the construction cost the emergency } \\
\text { medical service facility at point } j\end{array}$ \\
\hline$p_{j}$ & : & $\begin{array}{l}\text { the operational cost an emergency vehicle } \\
\text { at emergency medical service at point } j\end{array}$ \\
\hline$d_{i j}$ & : & $\begin{array}{l}\text { the distance between demand at point } i \\
\text { with points of candidate facility at point } j\end{array}$ \\
\hline$c$ & : & the unit transportation cost \\
\hline$q_{i}$ & : & $\begin{array}{l}\text { the maximum number of concurrent } \\
\text { demand at demand point } i\end{array}$ \\
\hline$\mu_{i}$ & : & the average demand at demand at point $i$ \\
\hline$M$ & : & sufficiently large number \\
\hline$W$ & : & the weight because unfilled demand \\
\hline
\end{tabular}

Decisions Variables are the following

$y_{j} \quad$ : equals 1 , if candidate facility $j$ open and 0 otherwise

$x_{i j} \quad$ : the proportion of demand point $i$ served by facility $j$

$n_{j} \quad$ : the number of emergency vehicles at facility $j$

Refers to Zhang and Jiang [1] the deterministic model of the bi-objective problem of emergency health service design that minimizes total costs and underserved requests within the specified travel distance is modelled as follows. The first objective function is to minimize the cost of building emergency health care facilities, emergency vehicle operating costs, and transportation costs from facilities to the point of request. 
$f_{1}: \min \sum_{j \in J} f_{j} y_{j}+\sum_{j \in J} p_{j} n_{j}+\sum_{j \in J} \sum_{i \in I} c d_{i j} \mu_{i} x_{i j}$

The second objective function is to minimize the demand that is not served in $S$ where the proposed objective function is to minimize the cost of building facilities, the cost of operating emergency vehicles and the cost of penalty weights given due to underserved requests within the $S$, travel distance.

$f_{2}: \min \sum_{j \in J} f_{j} y_{j}+\sum_{j \in J} p_{j} n_{j}+W \sum_{j \in J} \sum_{i \in I} \mu_{i} x_{i j}$

The constraint function (3) ensure that demand at each demand point are served

$\sum_{j \in J} x_{i j}=1, \forall i \in I$

The constraint function (4) and (5) state that demand point and vehicle can only be assigned to open EMS facility

$x_{i j} \leq y_{j}, \forall i \in I, j \in J$

$n_{j} \leq M y_{j}, \forall j \in J$

The constraint function (6) stipulate that the number of emergency vehicles at point $j$ is no less than maximum number of concurrent demands at demand point $i$.

$\sum_{i \in I} q_{i} x_{i j} \leq n_{j}, \forall j \in J$

Constraint (7) show the proportion of demand at location $i$ that can be served by EMS facility $j$

$0 \leq x_{i j} \leq 1, \forall i \in I, j \in J$

Constraint (8) state if facility is built then the value is 1 and otherwise 0 .

$y_{j} \in\{0,1\}, \forall j \in J$

Constraint (9) state the number of emergency vehicles that allocated to the built facility is integer value.

$n_{j} \in Z_{+}, \forall j \in J$

Thus, the complete optimization model for Bi-objective Problem of Emergency Medical Service Design can be rewrite as follows

$\min f_{1}:(1)$

$\min f_{2}:(2)$

s.t (3) - (9)

In the next section, discussion about the theory of Robust Optimization is presented. This theory is proposed by [15] and also discussed in [21], [22], [19] and also [20].

\section{Robust Optimization}

Based on [15], Uncertain Linear Programming (ULP) is defined as follows.

$\min _{x}\left\{c^{T} x: A x \leq b,(c, A, b) \in \mathcal{U}\right\}$
Where $c \in R^{n}, A \in R^{m \times n}$, and $b \in R^{m}$ denote uncertain coefficients, and $\mathcal{U}$ denotes uncertainty set. The decision environment is assumed to be in the following three conditions. Firstly, decisions are off-line the entire decision vector $x$ is to be fixed prior to knowing which value the actual parameters take ("here and now" decision). In the dynamic (ULP) case only part of the variables $\left(x_{1}, \ldots, x_{n}\right)$ need to be determined offline. The rest may be determined after some of the uncertain parameters become know ("wait and see" decision). Secondly, the information on the data $(c, A, b)$ is "crude" and is captured by a compact uncertainty set $\mathcal{U}$.Thirdly, the inequality constraint $A x \leq b$ are "hard": i.e, they must all be satisfied whenever the uncertain parameter resides in $\mathcal{U}$.

Robust optimization approach converts the uncertain problem of (11) into the following single deterministic problem, which call Robust Counterpart (RC):

$\pi^{*}=\min _{x}\left\{c^{T} x \mid A x \leq b, \forall(c, A, b) \in \mathcal{U}\right\}$

A vector $x^{*}$ is called a robust optimal solution if for all realization $(c, A, b) \in \mathcal{U}, x^{*}$ is feasible, and the value of the objective function is guaranteed to be at most $\pi^{*}$. Problem (RC) equivalent as a problem with a linear certain objective function and only uncertain constraints as follows:

$\min _{x, t}\left\{t: c^{T} x-t \leq 0, a_{i}^{T} x-b_{i} \leq 0, i=1, \ldots, m, \forall(c, A, b) \in\right.$ $x, t$
$u\}$

Note this problem is a semi-infinite programming problem.

In robust optimization, robustness with respect to $\mathcal{U}$ can be formulated constraint-wise, so that one can look at the impact of uncertainty on each constraint individually. At the risk of some ambiguity, we temporarily drop the constraint index $i$ and consider the canonical robust semi-infinite constraint

$a^{T} x-b \leq 0, \forall a \in \mathcal{U}$

Then, describe the uncertainty parameters $a$ and the uncertainty set $\mathcal{U}$ in term of a primitive factor $\zeta \in R^{L}$ which can be written as follows:

$a=\bar{a}+P \zeta$

Where $\bar{a} \in R^{L}, P \in R^{m \times L}$, and

$\mathcal{U}=\{(a=\bar{a}+P \zeta) \mid \zeta \in Z\}$

Where $Z \subset R^{L}$ is the uncertainty set for the primitive factor. The fixed vector $\bar{a}$ is called as nominal.

Based on [20] to produce a fulfilling representing $\mathcal{U}$ (17) can be defined the uncertainty $a$ in a simple interval as follows:

$\mathcal{U}=\left\{a \mid a^{l} \leq a \leq a^{u}\right\}$ 
With define:

$\bar{a}=\frac{a^{l}+a^{u}}{2}, P=\operatorname{diag}\left(\frac{a^{u}-a^{l}}{2}\right)$

The challenge in Robust Optimization is to find for which types of uncertainty sets problem can be reformulated into a tractable optimization problem, which is define as convex optimization problem (see [23]). According to [19] there are some uncertainty set including box uncertainty set and ellipsoidal uncertainty set which guarantee a computationally tractable robust counterpart can be achieved, as can be seen on [15] and [21].

\section{Box Uncertainty Set and Ellipsoidal Uncertain- ty Set}

Based on [20], in this section a derivation of a tractable RC formulation for robust linear optimization problem with interval (box) is presented. Since one of the assumptions is that the uncertainty is constraintwise, then

$(\bar{a}+P \zeta)^{T} x \leq b, \forall \zeta:\|\zeta\|_{\infty} \leq \rho$

Where $x$ satisfied (18) if and only if $x$ satisfies:

$\bar{a}^{T} x+\left\|P^{T} x\right\|_{1} \leq b$

According to [22], a special case of box uncertaity occurs when the uncertaity is relative to the nominal velues, so that the uncertainty set has the form

$\mathcal{U}=\{a: \mid a-\bar{a} \leq \gamma \bar{a}\}=[(1-\gamma) \bar{a},(1+\gamma) \bar{a}]$

Then we have:

$\max _{a \in \mathcal{U}} a^{T} x=((1+\gamma) \bar{a})^{T} x$

Choose $P=\gamma \bar{a}$ then:

$\left\|P^{T} x\right\|_{1}=\left\|(\gamma \bar{a})^{T} x\right\|_{1}=\left|(\gamma \bar{a})^{T} x\right|=(\gamma \bar{a})^{T} x$

In ellipsoidal uncertainty, Robust Counterpart becomes:

$(\bar{a}+P \zeta)^{T} x \leq b, \forall \zeta:\|\zeta\|_{2} \leq \rho$

where $x$ satisfies (23) if and only if $x$ satisfies: $\bar{a}^{T} x+\left\|P^{T} x\right\|_{2} \leq b$

In the next section the determination of Robust Optimization Model for Bi-objective Emergency Medical Service Design Problem with Demand Uncertainty is presented.

\section{Results and Discussions}

In this section the derivation of $\mathrm{RC}$ for uncertain $\mathrm{Bi}$ objective Emergency Medical Service Design Problem Model is presented. Refers to [1] assume that demand $\left(\mu_{\mathrm{i}}\right)$ and $\left(\mathrm{q}_{\mathrm{i}}\right)$ as uncertain parameters that effect in two objective function on the model become uncertain, so it must be conducted into certain objective function by eliminating the uncertain parameters from objecttive function and presenting it in form single variable $\tau_{1}$ and $\tau_{2}$.

Consider that the canonical form of Bi-objective Emergency Medical Service Design Problem Model (BEMSDPM) with demand uncertainty is as follows. $\min \tau_{1}$

$\min \tau_{2}$

s.t

$\sum_{j \in J} f_{j} y_{j}+\sum_{j \in J} p_{j} n_{j}+\sum_{j \in J} \sum_{i \in I} c d_{i j} \mu_{i} x_{i j}-\tau_{1}+\beta_{1}=0$

$\sum_{j \in J} f_{j} y_{j}+\sum_{j \in J} p_{j} n_{j}+W \sum_{j \in J} \sum_{i \in I} \mu_{i} x_{i j}-\tau_{2}+\beta_{2}=0$

$\sum_{j \in J} x_{i j}+r_{i}=1, \forall i \in I$

$x_{i j}+S_{i j}=y_{j}, \forall i \in I, j \in J$

$n_{j}+t_{j}=M y_{j}, \forall j \in J$

$\sum_{i \in I} q_{i} x_{i j}+u_{j} \leq n_{j}, \forall j \in J$

$x_{i j}+v_{i j}=1, \forall i \in I, j \in J$

$y_{j} \in\{0,1\}, \forall j \in J$

$n_{j} \in Z_{+}, \forall j \in J$

$r_{i} \geq 0, \forall i \in I$

$t_{j}, u_{j} \geq 0, \forall j \in J$

$x_{i j}, s_{i j}, v_{i j} \geq 0, \forall i \in I, j \in J$

$\tau_{1}, \tau_{2}, \beta_{1}, \beta_{2} \geq 0$

$\forall \mu_{i}, q_{i} \in \mathcal{U}, \forall i \in I$

Notes that $r_{i}, t_{j}, u_{j}, s_{i j}, v_{i j}, \beta_{1}, \beta_{2} \geq 0$ are slack variable that is added to get the canonical form.

The next step is to determine Robust Counterpart of uncertain model above. The formulation of Robust Counterpart (RC) depends on uncertainty set selected. If the right uncertainty set is selected, then Robust Counterpart can be formulated into tractable optimization problem. In the following section, the RC is presented in two cases of uncertainty sets, i.e., box and ellipsoidal uncertainty sets.

\section{Determining Robust Optimization Model with Box Uncertainty Set}

In case box uncertainty set, demand is assumed uncertain and bounded on a symmetry interval around certain nominal value, so the form of uncertainty set for this problem as follows.

$\mathcal{U}_{\mu, b o x}=\left\{\bar{\mu}+P \zeta:\|\zeta\|_{\infty} \leq 1\right\}$

$\mathcal{U}_{q, b o x}=\left\{\bar{q}+P \zeta:\|\zeta\|_{\infty} \leq 1\right\}$

where $\bar{\mu}, \bar{q} \in R^{n}$ are the nominal value, $P \in R^{n \times L}$ and $\zeta \in R^{L}$ is a primitive factor.

In this problem, the constraints with uncertain parameter are (27), (28), and (32). Assume that the uncertainty lies within a box uncertainty set (41), thus the constraint (27) can be re-write as follows. 
$\sum_{j \in J} f_{j} y_{j}+\sum_{j \in J} p_{j} n_{j}+\sum_{j \in J} \sum_{i \in I} c d_{i j} \mu_{i} x_{i j}-\tau_{1}+\beta_{1}, \forall \mu_{i} \in \mathcal{U}$

$\Leftrightarrow \sum_{j \in J} f_{j} y_{j}+\sum_{j \in J} p_{j} n_{j}+\max _{\mu \in \mathcal{U}_{\mu, b o x}} \sum_{j \in J} \sum_{i \in I} c d_{i j} \mu_{i} x_{i j}-\tau_{1}+\beta_{1}=0$

$\Leftrightarrow \sum_{j \in J} f_{j} y_{j}+\sum_{j \in J} p_{j} n_{j}+\max _{\|\zeta\|_{\infty} \leq 1} \sum_{j \in J} \sum_{i \in I} c d_{i j}\left(\bar{\mu}_{i}+\left(P_{i}\right)_{\mu}(\zeta)_{i}\right) x_{i j}-\tau_{1}+\beta_{1}=0$

$\Leftrightarrow \sum_{j \in J} f_{j} y_{j}+\sum_{j \in J} p_{j} n_{j}+\sum_{j \in J} \sum_{i \in I} c d_{i j} \bar{\mu}_{i} x_{i j}+\max _{\zeta:\|\zeta\|_{\infty} \leq 1} \sum_{j \in J} \sum_{i \in I} c d_{i j}\left(\left(P_{i}\right)_{\mu}(\zeta)_{i}\right) x_{i j}-\tau_{1}+\beta_{1}=0$

$\Leftrightarrow \sum_{j \in J} f_{j} y_{j}+\sum_{j \in J} p_{j} n_{j}+\sum_{j \in J} \sum_{i \in I} c d_{i j} \bar{\mu}_{i} x_{i j}+\sum_{j \in J} \sum_{i \in I}\left|c d_{i j}\left(P_{i}\right)_{\mu} x_{i j}\right|-\tau_{1}+\beta_{1}=0$

$\Leftrightarrow \sum_{j \in J} f_{j} y_{j}+\sum_{j \in J} p_{j} n_{j}+\sum_{j \in J} \sum_{i \in I} c d_{i j} \bar{\mu}_{i} x_{i j}+\left\|\sum_{j \in J} \sum_{i \in I} c d_{i j}\left(P_{i}\right)_{\mu} x_{i j}\right\|-\tau_{1}+\beta_{1}=0$

$\Leftrightarrow \sum_{j \in J} f_{j} y_{j}+\sum_{j \in J} p_{j} n_{j}+\sum_{j \in J} \sum_{i \in I} c d_{i j} \bar{\mu}_{i} x_{i j}+\sum_{j \in J} \sum_{i \in I} c d_{i j}\left(P_{i}\right)_{\mu} x_{i j}-\tau_{1}+\beta_{1}=0$

Using (22) thus the following formulation is obtained.

$\Leftrightarrow \sum_{j \in J} f_{j} y_{j}+\sum_{j \in J} p_{j} n_{j}+\sum_{j \in J} \sum_{i \in I} c d_{i j} \bar{\mu}_{i} x_{i j}+\sum_{j \in J} \sum_{i \in I} \gamma c d_{i j} \bar{\mu}_{i} x_{i j}-\tau_{1}+\beta_{1}=0$

Using the same techniques to constraint (28) and (32) as we do for obtaining the robust counterpart of constraint (27), with using box uncertainty set approach of (40) and (41) we have the equivalent form of (28) and (32) respectively as follows:

$\sum_{j \in J} f_{j} y_{j}+\sum_{j \in J} p_{j} n_{j}+$

$W \sum_{j \in J} \sum_{i \in I} \bar{\mu}_{i} x_{i j}+W \sum_{j \in J} \sum_{i \in I} \gamma \bar{\mu}_{i} x_{i j}-\tau_{2}+\beta_{2}=0$

$\sum_{i \in I} \bar{q}_{i} x_{i j}+\sum_{i \in I} \gamma \bar{q}_{i} x_{i j}-n_{j}+u_{j}=0$

Change constraint (27), (28) and (32) with constraint (43), (44) and (45) so we have the Robust Counterpart for BEMSDPM with demand uncertainty using box uncertainty set approach as follows :

$\min \tau_{1}$

$\min \tau_{2}$

s.t

$\sum_{j \in J} f_{j} y_{j}+\sum_{j \in J} p_{j} n_{j}+\sum_{j \in J} \sum_{i \in I} c d_{i j} \bar{\mu}_{i} x_{i j}+$

$\sum_{j \in J} \sum_{i \in I} \gamma c d \bar{\mu}_{i} x_{i j}-\tau_{1}+\beta_{1}=0$

$\sum_{j \in J} f_{j} y_{j}+\sum_{j \in J} p_{j} n_{j}+W \sum_{j \in J} \sum_{i \in I} \bar{\mu}_{i} x_{i j}+$

$W \sum_{j \in J} \sum_{i \in I} \gamma \bar{\mu}_{i} x_{i j}-\tau_{2}+\beta_{2}=0$

$\sum_{j \in J} x_{i j}+r_{i}=1, \forall i \in I$

$x_{i j}+S_{i j}=y_{j}, \forall i \in I, j \in J$

$n_{j}+t_{j}=M y_{j}, \forall j \in J$

$\sum_{i \in I} \bar{q}_{i} x_{i j}+\sum_{i \in I} \gamma \bar{q}_{i} x_{i j}-n_{j}+u_{j}=0$

$x_{i j}+v_{i j}=1, \forall i \in I, j \in J$

$y_{j} \in\{0,1\}, \forall j \in J$

$n_{j} \in Z_{+}, \forall j \in J$ $r_{i} \geq 0, \forall i \in I$

$t_{j}, u_{j} \geq 0, \forall j \in J$

$x_{i j}, s_{i j}, v_{i j} \geq 0, \forall i \in I, j \in J$

$\tau_{1}, \tau_{2}, \beta_{1}, \beta_{2} \geq 0$

Robust Counterpart Model (46) can we classify into

Linier Programing. So, Robust Counterpart can be guaranteed computationally tractable based on theorem in [15] and [21].

\section{Robust Optimization Model with Ellipsoidal Uncertainty Set Approach}

In case ellipsoidal uncertainty set, demand is assumed uncertain and bounded on a ball with radius $\Omega$ centered at the origin point, so the form of the uncertainty set for this problem as follows:

$\mathcal{U}_{\mu, e l l}=\left\{\bar{\mu}+P \zeta:\|\zeta\|_{2} \leq 1\right\}$

$\mathcal{U}_{q, e l l}=\left\{\bar{q}+P \zeta:\|\zeta\|_{2} \leq 1\right\}$

where $\bar{\mu}, \bar{q} \in R^{n}$ are the nominal value, $P \in R^{n \times L}$ and $\zeta \in R^{L}$ is a primitive factor. Constraint functions that have uncertainty parameter is (27), (28) and (32), so with using ellipsoidal uncertainty set approach in (46), in constraint (27) we have the equivalent form as follows:

$\sum_{j \in J} f_{j} y_{j}+\sum_{j \in J} p_{j} n_{j}+\sum_{j \in J} \sum_{i \in I} c d_{i j} \mu_{i} x_{i j}-\tau_{1}+\beta_{1}=0, \forall \mu_{i} \epsilon \mathcal{U}$

$\Leftrightarrow \sum_{j \in J} f_{j} y_{j}+\sum_{j \in J} p_{j} n_{j}+\max _{\mu \in \mathcal{U}_{\mu, e l l}} \sum_{j \in J} \sum_{i \in I} c d_{i j} \mu_{i} x_{i j}-\tau_{1}+\beta_{1}=0$

$\Leftrightarrow \sum_{j \in J} f_{j} y_{j}+\sum_{j \in J} p_{j} n_{j}+\max _{|\zeta|_{2} \leq 1} \sum_{j \in J} \sum_{i \in I} c d_{i j}\left(\bar{\mu}_{i}+\left(P_{i}\right)_{\mu}(\zeta)_{i}\right) x_{i j}-\tau_{1}+\beta_{1}=0$

$\Leftrightarrow \sum_{j \in J} f_{j} y_{j}+\sum_{j \in J} p_{j} n_{j}+\sum_{j \in J} \sum_{i \in I} c d_{i j} \bar{\mu}_{i} x_{i j}+\max _{|\zeta|_{2} \leq 1} \sum_{j \in J} \sum_{i \in I} c d_{i j}\left(\left(P_{i}\right)_{\mu}(\zeta)_{i}\right) x_{i j}-\tau_{1}+\beta_{1}=0$

$\Leftrightarrow \sum_{j \in J} f_{j} y_{j}+\sum_{j \in J} p_{j} n_{j}+\sum_{j \in J} \sum_{i \in I} c d_{i j} \bar{\mu}_{i} x_{i j}+\left\|\sum_{j \in J} \sum_{i \in I} c d_{i j}\left(P_{i}\right)_{\mu} x_{i j}\right\|_{2}-\tau_{1}+\beta_{1}=0$

Using (23) and (24) the following equation is obtained.

$\Leftrightarrow \sum_{j \in J} f_{j} y_{j}+\sum_{j \in J} p_{j} n_{j}+\sum_{j \in J} \sum_{i \in I} c d_{i j} \bar{\mu}_{i} x_{i j}+\sqrt{\sum_{j \in J} \sum_{i \in I}\left(c d_{i j}\left(P_{i}\right)_{\mu} x_{i j}\right)^{2}}-\tau_{1}+\beta_{1}=0$

Using the same techniques to constraint (28) and (32) as we do for obtaining the robust counterpart of constraint (27), with using ellipsoidal uncertainty set approach on (47) and (48) we have the equivalent form of (27) and (32) respectively as follows: 


$$
\begin{aligned}
& \sum_{j \in J} f_{j} y_{j}+\sum_{j \in J} p_{j} n_{j}+W \sum_{j \in J} \sum_{i \in I} \bar{\mu}_{i} x_{i j}+W \sqrt{\sum_{j \in J} \sum_{i \in I}\left(\left(P_{i}\right)_{\mu} x_{i j}\right)^{2}}-\tau_{2}+\beta_{2}=0 \\
& \sum_{i \in I} \bar{q}_{i} x_{i j}+\sqrt{\sum_{i \in I}\left(\left(P_{i}\right)_{\mu} x_{i j}\right)^{2}}-n_{j}+u_{j}=0
\end{aligned}
$$

Change constraint (27), (28) and (32) with constraint (49), (50) and (51), so we have the Robust Counterpart for BEMSDPM with demand uncertainty using ellipsoidal uncertainty set approach as follows:

$$
\begin{aligned}
& \min \tau_{1} \\
& \min \tau_{2} \\
& \text { s.t } \\
& \sum_{j \in J} f_{j} y_{j}+\sum_{j \in J} p_{j} n_{j}+\sum_{j \in J} \sum_{i \in I} c d_{i j} \bar{\mu}_{i} x_{i j}+ \\
& \sqrt{\sum_{j \in J} \sum_{i \in I}\left(c d_{i j}\left(P_{i}\right)_{\mu} x_{i j}\right)^{2}}-\tau_{1}+\beta_{1}=0 \\
& \sum_{j \in J} f_{j} y_{j}+\sum_{j \in J} p_{j} n_{j}+W \sum_{j \in J} \sum_{i \in I} \bar{\mu}_{i} x_{i j}+ \\
& W \sqrt{\sum_{j \in J} \sum_{i \in I}\left(\left(P_{i}\right)_{\mu} x_{i j}\right)^{2}}-\tau_{2}+\beta_{2}=0 \\
& \sum_{j \in J} x_{i j}+r_{i}=1, \forall i \in I \\
& n_{j}+t_{j}=M y_{j}, \forall j \in J \\
& \sum_{i \in I} \bar{q}_{i} x_{i j}+\sum_{i \in I} \gamma \bar{q}_{i} x_{i j}-n_{j}+u_{j}=0 \\
& x_{i j}+v_{i j}=1, \forall i \in I, j \in J \\
& y_{j} \in\{0,1\}, \forall j \in J \\
& n_{j} \in Z_{+}, \forall j \in J \\
& r_{i} \geq 0, \forall i \in I \\
& t_{j}, u_{j} \geq 0, \forall j \in J \\
& x_{i j}, s_{i j}, v_{i j} \geq 0, \forall i \in I, j \in J \\
& \tau_{1}, \tau_{2}, \beta_{1}, \beta_{2} \geq 0
\end{aligned}
$$

Robust Counterpart Model (52) can we classify into Conic Quadratic Optimization. So, Robust Counterpart can be guaranteed computationally tractable based on theorem in [15] and [21]. This means that Robust Counterpart Model (52) can be solved in polynomial time algorithm and the global optimal solution is guaranteed to be obtained.

In the next section an example is presented.

\section{Example}

In this section numerical experiments are conducted for the BEMSDPM. In the numerical experiment, the location of health facilities will be determined in several predetermined location candidates. Then the number of emergency vehicles will be determined in the facilities built, as well as the proportion of requests served by the facility. Cases used are cases of secondary data sourced from journals written by Ndiaye and Alfares [18].

Ndiaye and Alfares [18] discuss the determination of the location of health facilities with requests that depend on summer and cold with case studies covering 10 locations of health facilities and 17 locations of demand. In this numerical experiment, the data used is demand data in the summer with three health facility location points and five demand location points. Besides, the data used is the distance of health facilities to the point of request, the cost of building facilities, and the average demand.

The data used in this numerical experiment is as follows: the constructed facility is a health care facility. In this case, is assumed the number of constructed facility location candidate is 3 location and demand points are 5 locations. The operational cost to allocation emergency vehicle in facility $j=$ 1,2,3 respectively are 110,000,56,000, 130,000 The unit of transportation cost is assumed 50 . The maximum number of concurrent demand at demand points $i=1,2,3,4,5$ respectively are 90,19,39,183,103. A significant amount in this problem is defined as the number of vehicles capable of meeting all requests that will be returned by all health service facilities. In this problem, assume that a fairly large number with a value. The maximal travel distance to measure service level from all medical facility is 100 distance unit. The weight because not served is 50. The constructed facility cost, the distance between health care facility and demand points, and average demand at demand points according to research [18] with data are used in the summer season with cost 115,700, 125,000 and 270,000 .

For calculation the box uncertainty (44) and (45) also the ellipsoidal (47) and (48), use formulation (16) amd (17) thus the parameter $\overline{\mu_{l}}, \bar{q}_{l},\left(P_{i}\right)_{\mu}$, and $\left(P_{i}\right)_{q}$ are obtained as can be seen in Table 1 and Table 2 .

Table 1. The result $\bar{\mu}_{\iota}$ and $\left(P_{i}\right)_{\mu}$

\begin{tabular}{cccll}
\hline$i$ & $\mu_{i}^{l}$ & $\mu_{i}^{u}$ & \multicolumn{1}{c}{$\left(P_{i}\right)_{\mu}$} & \multicolumn{1}{c}{$\bar{\mu}_{i}$} \\
\hline 1 & 337 & 525 & 94 & 431 \\
2 & 337 & 337 & 0 & 337 \\
3 & 337 & 594 & 128,5 & 465,5 \\
4 & 337 & 542 & 102,5 & 439,5 \\
5 & 337 & 530 & 96,5 & 433,5 \\
\hline
\end{tabular}

Table 2. Value result $q_{i}$ and $\left(P_{i}\right)_{q}$

\begin{tabular}{cclll}
\hline$i$ & $q_{i}^{l}$ & \multicolumn{1}{c}{$q_{i}^{u}$} & \multicolumn{1}{c}{$\left(P_{i}\right)_{q}$} & \multicolumn{1}{c}{$q_{i}$} \\
\hline 1 & 19 & 90 & 35,5 & 54,5 \\
2 & 19 & 19 & 0 & 19 \\
3 & 19 & 29 & 10 & 29 \\
4 & 19 & 183 & 82 & 101 \\
5 & 19 & 103 & 42 & 61 \\
\hline
\end{tabular}


The data has been obtained then substituted to a nominal bi-objective emergency medical service design problem (1)-(9). For calculation in this example for the box uncertainty (45) is chosen with, to show there is some variety of the data on the box uncertainty.

The optimal solution can be seen in Table 3 , where the results show that for the nominal model (1)-(9)., RC with box uncertainty set (46) and RC with ellipsoidal uncertainty set (52). The bi-objective functions are done using the Lexicographic Methods where the ranks objective functions based on priority (see [16]) and the integer binary variables are handled using Branch and Bound Methods (see [17]).

The models show that facility is built at the same points i.e. location $j=2$. The optimal solution obtained also shows that emergency vehicles are allocated to health facilities on location $j=2$, but the difference in each model only the number of emergency vehicles at the facility.

In terms of robustness, the robust formulation counterpart of the BEMSDPM uncertain optimization problem is an optimization problem with a computationally tractable model. The model is a linear optimization and conic quadratic optimization. Some literature (see Ben-Tal and Nemirovskii [15] and Gorissen et al. [19]) stated that with this box selection and ellipsoidal uncertainty set, the uncertain problem of BEMSDPM no longer contains semiinfinite problem classes. Moreover, the RC formulations as linear and conic quadratic optimization are needed to ensure the existence of global optimal solutions. So that the critical issue to obtain an RC formulation that is computationally tractable has been fulfilled.

Furthermore, because this problem includes a set covering problem where all requests must be served, all requests are only served by health facilities at location $j=2$. Because demand at point $i$ can only be served by health facilities built at location $j$, then it will be shown that the calculation results fulfill the statement. The total number of vehicles needed in location $j=2$ for the nominal model, $\mathrm{RC}$ with box and RC with ellipsoidal uncertainty are 434, 478 and 367 vehicle. This number depends on the amount of $M$, the number of vehicles capable of meeting all requests that will be returned by all health service facilities. In this problem, assume that a fairly large number with a value $M \geq 500$ otherwise the model found the condition of no feasible point found for ILP subproblem when solving the nominal model.
Table 3. Optimal solution to the examples

\begin{tabular}{|c|c|c|c|}
\hline $\begin{array}{l}\text { Decision } \\
\text { variable }\end{array}$ & $\begin{array}{l}\text { Nominal } \\
\text { model }\end{array}$ & $\begin{array}{l}\text { RC Model } \\
\text { with box } \\
\text { uncertainty } \\
\text { set approach } \\
\gamma=10 \%\end{array}$ & $\begin{array}{l}\text { RC Model } \\
\text { with } \\
\text { ellipsoidal } \\
\text { uncertainty } \\
\text { set approach }\end{array}$ \\
\hline$x_{1,1}$ & 0 & 0 & 0 \\
\hline$x_{1,2}$ & 1 & 1 & 1 \\
\hline$x_{1,3}$ & 0 & 0 & 0 \\
\hline$x_{2,1}$ & 0 & 0 & 0 \\
\hline$x_{2,2}$ & 1 & 1 & 1 \\
\hline$x_{2,3}$ & 0 & 0 & 0 \\
\hline$x_{3,1}$ & 0 & 0 & 0 \\
\hline$x_{3,2}$ & 1 & 1 & 1 \\
\hline$x_{3,3}$ & 0 & 0 & 0 \\
\hline$x_{4,1}$ & 0 & 0 & 0 \\
\hline$x_{4,2}$ & 1 & 1 & 1 \\
\hline$x_{4,3}$ & 0 & 0 & 0 \\
\hline$x_{5,1}$ & 0 & 0 & 0 \\
\hline$x_{5,2}$ & 1 & 1 & 1 \\
\hline$x_{5,3}$ & 0 & 0 & 0 \\
\hline$n_{1}$ & 0 & 0 & 0 \\
\hline$n_{2}$ & 434 & 478 & 367 \\
\hline$n_{3}$ & 0 & 0 & 0 \\
\hline$y_{1}$ & 0 & 0 & 0 \\
\hline$y_{2}$ & 1 & 1 & 1 \\
\hline$y_{3}$ & 0 & 0 & 0 \\
\hline$\tau_{1}^{*}$ & $40.051 .440,50$ & $44.077 .684,55$ & $35.063 .083,52$ \\
\hline$\tau_{2}^{*}$ & 24.482 .600 & 26.951.959,99 & 20.733.927,33 \\
\hline
\end{tabular}

\section{Conclusions}

In bi-objective emergency medical service design problem according to [1] has the demand parameter, where the demand parameter is uncertain. In handling the uncertain demand, Robust Optimization approach is employed. By assuming the uncertainty lies on a box and ellipsoidal uncertainty set. the computational tractability of the Robust Counterpart can be obtained, and the RC becomes Linear Programming and Conic Quadratic Programming. This means that the $\mathrm{RC}$ is guaranteed to have an optimal global solution. As future research, the Model Emergency Health Service Design Bi-Objective Problem proposed by Zhang and Jiang in [1] with uncertainty on demand can be developed with the new approach of Robust Optimization Model using the new uncertainty set approach such as the polyhedral uncertainty set (see [19] and [20]). Then, Robust Optimization Model of Bi-Objective Problems Emergency Health Service Design with Request Uncertainty can be applied in determining the location of new health 
centers with a case study of Kebumen District, and Central Java sees Mahanani, and Rahardjo in [3].

\section{Acknowledgement}

This research is funded by Penelitian Berbasis Kompetensi Kemenristekdikti for the year of 2018 under contract number 1075/UN6.D/LT/2018

\section{References}

1. Zhang, Z.H., and Jiang, H., A Robust Counterpart Approach to The Bi-Objective Emergency Medical Service Design Problem, Applied Mathematical Modelling, 38(3), 2014, pp. 1033-1040.

2. KBBS, Kementerian Pendidikan dan Kebudayaan, Kamus Besar Bahasa Indonesia, 2016. [Online]. Available:

https://kbbi.kemdikbud.go.id. [Accessed 22 2018].

3. Mahanani, M. and Rahardjo, N., Penentuan Lokasi Puskesmas Baru Menggunakan Analitycal Hierarchy Process (Studi Kasus Kabupaten Kebumen, Jawa Tengah), Jurnal Bumi Indonesia, 4(3), 2015.

4. Fasilitas Pelayanan Kesehatan, Peraturan Pemerintah Republik Indonesia no 47 tahun 2016, [Online]. Available: https://www.bphn.go.id/data/ documents/16pp047.pdf. [Accessed 152 2018].

5. Church, R. and ReVelle, C., The Maximal Covering Location Problem, Paper of The Regional Science Association, 32(1),1974, pp. 101-118.

6. Toregas, C., Swain, R., Revelle, C., and Bergman, L., The Location of Emergency Service Facilities, Operation Research, 1363-1373, 1971.

7. Gendreu, M., Laporte, G., and Semet, F., Solving an Ambulance Location Model by Tabu Search, Location Science, 5(2), 1997, pp. 75-88.

8. Harewood, S. I., Emergency Ambulance Deployment in Barbados: A Multi-objective Approach, Journal of the Operational Research Society, 53(2), 2002, pp. 185-102.

9. Araz, C., Selim, H., and Ozkarahan, I., A Fuzzy Multi-objective Covering-Based Vehicle Location Model for Emergency Service, Computer \& Operations Research, 34(3), 2007, pp. 705-726, 2007.

10. Almeida, L. A., Tralhao, L., Santos, L. and Rodri, A Multiobjective Approach to Locate Emergency
Areas, Geographical Analysis, 4(1), 2009, pp. 9-29. 11. Moghaddam, R. T., Memari, P., and Talebi, E., A Bi-Objective Location-Allocation Problem of Temporary Emergency Stations and Ambulance Routing in a Disaster Situation, in IEEE 4th International Conference on Optimization and Applications (ICOA), pp. 1-4. doi: 10.1109/ICOA. 2018. 8370579, Mohammedia, Morocco, 2018.

12.Bai, X., Two-Stage Multiobjective Optimization for Emergency Supplies Allocation Problem under Integrated Uncertainty, Mathematical Problems in Engineering, 2016,pp. 1-13.

13. Beraldi, P., Bruni, M. E., and Conforti, D., Designing Robust Emergency Medical Service via Stochastic Programming, European Journal of Operation Research, 158(1), 2004, pp. 183-193.

14. Beraldi, P. and Bruni, M.E., A Probabilistic Model Aplied to Emergency Service Vehicle Location, European Journal of Operation Research, 196(1),2009, pp. 323-331.

15. Bental, A. and Nemirovskii, A., Robust Optimization - Methodology and Applications, Mathematical Programming, 92(3), 2002, pp. 453-480.

16. Rao, S. S., Enginering Optimization Theory and Practice, New Jersey: John Wiley \& Sons, Inc., 2009.

17. Hiller, F., and Lieberman, G., Introduction to Operations Research, New York: McGraw-Hill., 2010.

18. Ndiaye, M., and Alfares, H., Modeling Health Care Facility Location for Moving Population Group, Computers and Operation Research, 35(7), 2008, pp. 2154-2161.

19. Gorissen, B., Yamkoglu, I. and Hertog, D. d., A Practical Guide to Robust Optimization, Omega., 53, 2015, pp. 124-137.

20.Hertog, D. d., Practical Robust Optimization: Lecture Notes LNMB Course, Tilburg University The Netherlands, Tilburg, 2015.

21. Chaerani, D., and Roos, C. Handling Optimization under Uncertainty Problem Using Robust Counterpart Methodology, Jurnal Teknik Industri, 15(2), 2013, pp. 111-118.

22. Chaerani, D., Roos, C. and Aman, A., The Robust Shortest Path Problem by Means of Robust Linear Optimization) in Operations Research Proceedings, Tilburg, 2004.

23. Boyd, S., and Vandenberghe, L., Convex Optimization, Cambridge: Cambridge University Press, 2004. 
Chaerani et al. / Robust Optimization Model / JTI, Vol. 20, No. 2, December 2018, pp. 95-104 